\title{
PRELIMINARY PLUME CHARACTERIZATION OF A LOW-POWER HALL THRUSTER CLUSTER
}

\author{
Brian E. Beal ${ }^{*}$ and Alec D. Gallimore ${ }^{\dagger}$ \\ Plasmadynamics and Electric Propulsion Laboratory \\ Department of Aerospace Engineering \\ The University of Michigan \\ Ann Arbor, MI 48109 USA \\ William A. Hargus, Jr. ${ }^{*}$ \\ Air Force Research Laboratories \\ Edwards Air Force Base \\ Edwards, CA 93524 USA
}

\begin{abstract}
In an effort to understand the technical issues related to running multiple Hall effect thrusters in close proximity to each other, testing of a cluster of four Busek BHT-200-X3 devices has begun in Chamber 6 at the Air Force Research Laboratory. Preliminary measurements have shown that the variations in the discharge currents of the four thrusters are synchronized, possibly due to cross talk through the thruster plumes. Measurements of plasma density, electron temperature, and plasma potential in the thruster plumes obtained using a triple Langmuir probe are presented. Anomalously high electron temperatures were recorded along the centerline of each thruster. Collisionless, magnetosonic shock waves induced by the ion-ion two-stream instability are proposed as a possible cause of the high temperatures. The unperturbed ion velocity distribution along the centerline of a Hall thruster is shown to be unstable and a simple geometric model is presented to illustrate the qualitative changes in plasma properties expected across the proposed shock. Estimates using this model show that relatively large changes in electron temperature are consistent with small changes in electron number density across a shock. Qualitative arguments are presented indicating that collisionless shocks are unlikely to form as a result of clustering multiple thrusters.
\end{abstract}

\section{$\underline{\text { Introduction }}$}

Future Air Force plans foresee a need for electric propulsion systems capable of operating in the 100-150 kW power regime for use on orbit transfer vehicles and rescue vehicles capable of repositioning satellites that have exhausted their propellant load or failed to meet their operational orbit. ${ }^{1,2}$ The most viable type of electric propulsion device for this class of mission is the Hall thruster due to its low specific mass, high thrust density, and high reliability. Although the desired power level is well beyond the current state-ofthe-art, two approaches have been considered for reaching this power level.

The first method for reaching very high powers, known as the monolithic approach, is to design a single thruster capable of operating at the desired power level. NASA has pursued this approach through efforts to develop successively larger Hall thrusters.
That effort began with extensive testing of the Pratt \& Whitney T-220 $10 \mathrm{~kW}$ class engine and has now proceeded to the development of a $50 \mathrm{~kW}$ thruster. $^{3}$ The monolithic approach may prove problematic due to the lack of ground facilities capable of maintaining adequate test pressures while supporting the high mass flow rates required of a Hall thruster operating at power levels above $100 \mathrm{~kW}$.

The second method, which is the one favored by the United States Air Force, involves clustering several lower powered Hall thrusters. ${ }^{4}$ A cluster of thrusters may have a slightly lower efficiency and higher dry mass than a similarly powered monolithic thruster since larger thrusters have historically performed better than smaller thrusters. The clustered approach, however, does have several advantages, including improved system reliability due to the

\footnotetext{
* Graduate Student, Student Member AIAA

$\dagger$ Associate Professor, Associate Fellow AIAA

$\ddagger$ Research Scientist, Senior Member AIAA
} 
inherent redundancy of running multiple engines, as well as the ability to throttle the system by simply turning off one or more of the thrusters. Throttling the system in this way allows the cluster to operate at lower power without running any of the individual thrusters at off-design conditions. This aspect of a cluster may prove beneficial, for example, on a geosynchronous satellite where a high-power Hall thruster cluster could be used for the initial LEO-GEO transfer and one element of the cluster could then be used for northsouth station keeping (NSSK). An additional, and perhaps very important, advantage of clustering is the high degree of system scalability. This means that, in principle, once the technical issues involved with operating a cluster are fully understood, a single flightqualified engine can support a wide range of missions requiring various power levels by simply clustering the appropriate number of thrusters. Thus, enhanced scalability and flexibility make clusters attractive for many missions.

Although using a cluster of commercially available thrusters for primary propulsion appears to be advantageous for some missions, there are several issues that must be addressed before clusters can be used in flight. ${ }^{1,2}$ For example, the neutralization process must be examined to determine whether a single cathode can be used to neutralize the entire system. The possibility of thruster cross talk through the plasma plumes must be addressed to determine how the proximity of several thrusters affects cluster operation. Perhaps the most pressing issue is the need to understand the interaction of the plasma plumes with each other and with the spacecraft. It is important to understand how clustering affects the divergence of the plume and to learn how the plume properties of a cluster can be simulated based on knowledge of the plume of a single unit.

In an effort to study the issues mentioned above, testing of a cluster of four 200-watt Busek BHT200-X3 Hall thrusters has begun in Chamber 6 at the Air Force Research Laboratory (AFRL). Although clustering is being considered primarily to reach very high power levels, initial testing is being conducted on clusters of low-power thrusters to facilitate testing in existing vacuum facilities. Additionally, any adverse effects of clustering are expected to be most pronounced in small thrusters due to the higher plasma densities associated with these devices. Figures 1 and 2 show the cluster firing in Chamber 6.

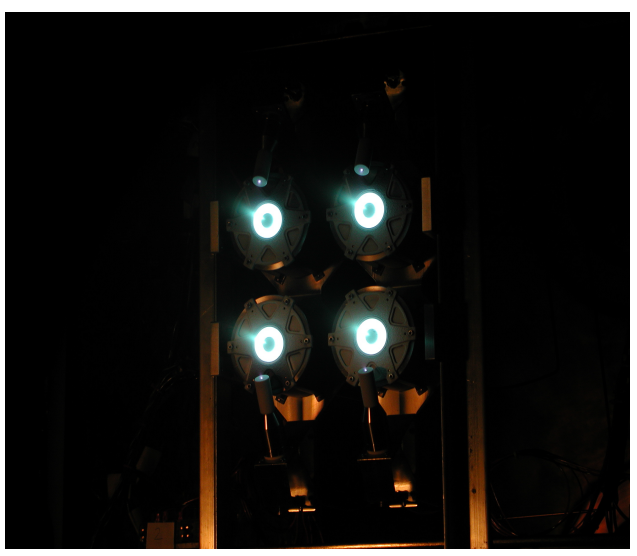

Figure 1: Low-power Hall thruster cluster operating in Chamber 6.

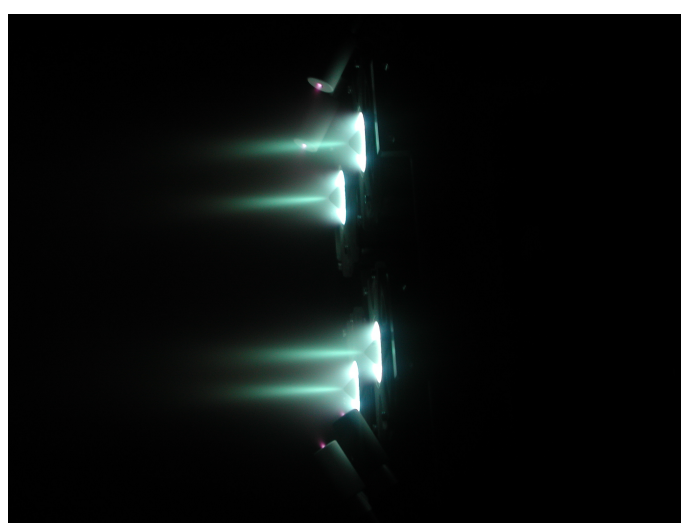

Figure 2: Side view of a cluster firing.

The plume of the cluster was characterized by an electrostatic triple probe, ${ }^{5}$ which was used to measure the electron number density, electron temperature, floating potential, and plasma potential as a function of position. The triple Langmuir probe and the closely related quadruple probe have been used successfully in studies of Hall effect thrusters, ${ }^{6}$ magnetoplasmadynamic (MPD) thrusters, ${ }^{7}$ and arcjets. ${ }^{8}$

\section{Experimental Setup}

\section{Cluster}

The cluster used in this experiment was composed of four Busek BHT-200-X3 200-watt class Hall thrusters. An earlier version of this thruster was reported to operate at an anode efficiency of $42 \%$ and specific impulse of 1300 seconds while providing 12.4 $\mathrm{mN}$ of thrust at the nominal operating conditions. ${ }^{9}$ Each thruster has a mean diameter of $21 \mathrm{~mm}$. The thrusters are arranged in a $2 \times 2$ grid with approximately 11.4 centimeters between the centerlines of adjacent thrusters. 
Each thruster in the cluster is completely independent of the others. Every thruster has its own Busek $3.2 \mathrm{~mm}$ diameter hollow cathode, whose voltage is allowed to float independently of the other three. In the future, experiments will be performed using a single cathode neutralizer and a single discharge power supply, however the work reported here was conducted with a completely modular system. Thruster operating conditions were monitored using an HP 34970A data acquisition system controlled by LabView software. All measured currents were converted to voltages via Hall sensors before being recorded by the data acquisition system.

Four power supplies are required to run each thruster. The main discharge supply for each thruster is a Sorensen DHP 400-5 digital power supply. Two Sorensen DLM 40-15 supplies are used to provide the heater and magnet currents to each device. The cathode is ignited by a DCS 600-1.7E. A UNIT Model UFC7301 mass flow controller calibrated for xenon regulates the propellant mass flow to each thruster and cathode individually.

\section{Vacuum Chamber}

All data reported in this paper were recorded in Chamber 6 at AFRL. Chamber 6 is a $1.5 \times 2.4$ meter cylindrical, stainless steel vacuum chamber that is evacuated by four cryopanels maintained at 25 Kelvin by four APD cold heads, HC-8C helium compressors, and an APD cryopump. ${ }^{10}$ This system provides a pumping speed of 26,000 liters per second of xenon with a base pressure of $8 \times 10^{-7}$ Torr as measured by a MKS Model 910 hot cathode gauge.

\section{Coordinate System}

The cluster installed in Chamber 6 is shown in Figure 3 where the thrusters are labeled as TH 1-4. Thruster 1 is in the top left-hand corner in Figure 3 and the numbering proceeds counterclockwise from there. Figure 3 also shows the coordinate system used in this experiment where $\mathrm{X}$ is to the right, $\mathrm{Y}$ is up, and $\mathrm{Z}$ is parallel to the thrust axis. The origin of the system is defined as the midpoint of the cluster in the X-Y plane and is located $50 \mathrm{~mm}$ downstream of the exit plane.

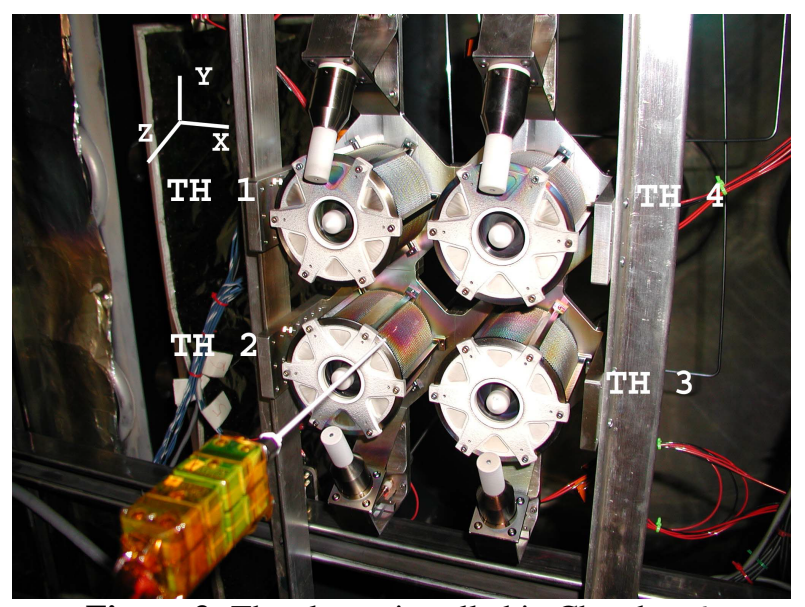

Figure 3: The cluster installed in Chamber 6.

\section{Positioning System}

Probes were swept through the cluster plume by a three-dimensional positioning system. The $X$ and $\mathrm{Z}$ positions were controlled by a single two-axis Parker Daedal table with approximately $30 \mathrm{~cm}$ of travel in each direction. The $\mathrm{Y}$ position was varied using a Parker Daedal linear stage having a $45 \mathrm{~cm}$ range of motion.

\section{Triple Probe}

The triple probe used for this experiment consisted of 3 tungsten electrodes insulated from each other by an alumina rod. The diameter of each electrode was $0.38 \mathrm{~mm}(0.015 ")$ and the length extending past the end of the alumina was $3.8 \mathrm{~mm}$ $(0.15$ "). A length to diameter ratio of 10 was chosen so that the area of the end of each electrode could be neglected compared to the area of the cylindrical surface. The spacing between the electrodes was approximately two electrode diameters. The probe was sized to criteria that allow the standard assumptions of probe theory to be applied. ${ }^{6}$ These criteria are summarized in Eqn. 1-5 below and are necessary to ensure that all ions entering the probe sheath are collected by the probe rather than being deflected by magnetic fields or collisions. Further, it is assumed that the electrodes are far enough apart to avoid interaction with each other and that the spatial gradients of plasma properties are sufficiently small such that all three electrodes are exposed to identical plasmas. Additionally, the electron energy distribution is assumed to be Maxwellian. In the relations that follow, $r_{L i, e}$ are the ion and electron Larmor radii, $r_{p}$ is the probe radius, $\lambda_{\mathrm{D}}$, is the Debye length, $\mathrm{dx}$ depicts the distance between adjacent electrodes, and $\lambda_{c}$ represents the total collision mean free path. The characteristic length scale over which plasma properties change significantly is denoted by $\Delta$. The data collected in this experiment were found to satisfy Eqn. 1-5 for the majority of the 
sampled volume. The small regions of the plume where the criteria given by Eqn. 2 and 4 are not met are discussed in subsequent sections of this paper.

$$
\begin{aligned}
& \mathrm{r}_{\mathrm{Li}}>>\mathrm{r}_{\mathrm{Le}}>>\lambda_{\mathrm{D}} \\
& \lambda_{\mathrm{D}}<<\mathrm{r}_{\mathrm{p}} \\
& \lambda_{\mathrm{c}}>>\mathrm{r}_{\mathrm{p}} \\
& \mathrm{dx}>>\lambda_{\mathrm{D}} \\
& \Delta>>\mathrm{r}_{\mathrm{p}}
\end{aligned}
$$

The symmetric triple probe, originally developed by Chen and Sekiguchi, ${ }^{5}$ is a convenient plasma diagnostic to use in Hall thruster plumes due to the elimination of the voltage sweep required by other electrostatic probes. Additionally, since the probe as a whole floats, the disturbance to the ambient plasma is minimized compared to single probes, which draw a net current from the discharge. A schematic of the triple probe is shown in Figure 4. In Figure 4, probe 2 is allowed to float while the voltage, $\mathrm{V}_{\mathrm{d} 3}$, is applied by a laboratory power supply with floating outputs. For the tests reported here, $\mathrm{V}_{\mathrm{d} 3}$ was set to 25 volts. The potential between probes 1 and $2, \mathrm{~V}_{\mathrm{d} 2}$, is measured by an HP 34970A data acquisition system, as are the floating potential, $\mathrm{V}_{\mathrm{f}}$, and the current, I. The probes are numbered in order of decreasing potential such that probe 2 is at the floating potential while probes 1 and 3 are biased above and below the floating potential, respectively.

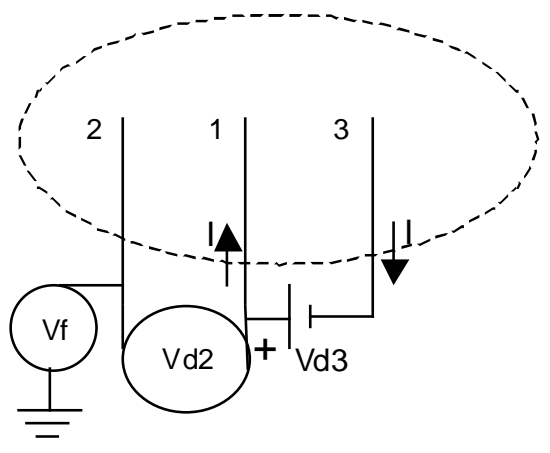

Figure 4: Triple probe circuit.

The relations used to determine plasma properties from measured probe data are presented in Eqn. 6-8. In these equations, $\mathrm{n}_{\mathrm{e}}$ is the electron number density, which is equal to the ion number density through the quasineutrality assumption. The electron temperature is represented by $\mathrm{T}_{\mathrm{e}}$, plasma potential by $\mathrm{V}_{\mathrm{p}}$, and ion and electron masses by $\mathrm{m}_{\mathrm{i}}$ and $\mathrm{m}_{\mathrm{e}}$, respectively. The symbol A denotes the area of a single electrode, $\mathrm{e}$ is the electron charge, and $\mathrm{k}_{\mathrm{b}}$ is Boltzmann's constant. Various error analyses indicate that the uncertainty in the calculated electron temperature and number density are generally less than $30 \%$ and $50 \%$, respectively. 5,7

$$
\begin{gathered}
n_{e}=\left(\frac{e m_{i}}{k_{b} T_{e}}\right)^{1 / 2} \frac{I \exp \left(\frac{1}{2}\right)}{A e^{3 / 2}\left[\exp \left(\frac{e V_{d 2}}{k_{b} T_{e}}\right)-1\right]} \\
\frac{1-\exp \left(\frac{-e V_{d 2}}{k_{b} T_{e}}\right)}{1-\exp \left(\frac{-e V_{d 3}}{k_{b} T_{e}}\right)^{2}}=\frac{1}{2} \\
V_{p}=V_{f}+\left|\left(\frac{k_{b} T_{e}}{e}\right) \ln \left[\left(\frac{2 \pi m_{e}}{m_{i}}\right)^{1 / 2} \exp (-1 / 2)\right]\right|
\end{gathered}
$$

\section{$\underline{\text { Experimental Results }}$}

\section{Thruster Operation}

Thruster operating conditions were recorded at approximately 25-second intervals. The discharge voltage, propellant mass flow, and electromagnet current were identical for each thruster. Typical thruster operating conditions are summarized in Table 1. When all four thrusters were operating at the nominal conditions, the pressure in the chamber rose to $6.4 \times 10^{-5}$ Torr indicated by the hot cathode gauge, which is calibrated on air.

\begin{tabular}{|c|c|}
\hline Parameter & Value \\
\hline Discharge Voltage (V) & $250 \pm 0.5$ \\
\hline Discharge Current (A) & $0.85 \pm 0.03$ \\
\hline Cathode Voltage (V) & $-8.5 \pm 1.0$ \\
\hline Magnet Current (A) & $1.0 \pm 0.03$ \\
\hline Keeper Current (A) & $0.5 \pm 0.05$ \\
\hline Keeper Voltage (V) & $13 \pm 1$ \\
\hline Anode Mass Flow (sccm) & $8.5 \pm 0.85$ \\
\hline Cathode Mass Flow (sccm) & $1.0 \pm 0.1$ \\
\hline
\end{tabular}

Table 1: Typical thruster operating conditions.

The measured discharge current and cathode potential with respect to ground for each thruster are displayed in Figure 5. This figure shows several interesting features, starting with the slightly different 
values of discharge current and cathode potential for each thruster. The variations between the two parameters do not appear to be correlated in any way and there is no clear trend in either measurement as a function of time.

Another interesting feature shown by the thruster discharge currents is the sudden increase in discharge current variations as a function of time. Figure 6 shows a portion of the discharge current trace from Figure 5. The fact that the discharge currents fluctuate somewhat over time is not surprising since that is a property of many Hall thrusters. The variations are less than $2 \%$ of the steady state value and are not considered unusual. The interesting feature shown in Figure 6, however, is the synchronization of the discharge current variations. The discharge currents all seem to increase and decrease in unison and the magnitude of the variations is consistent from thruster to thruster. Although there is no reason to suspect that the performance of the individual thrusters has been compromised, the data do seem to indicate that the thrusters are interacting with each other. Since the power supplies and propellant feed systems of each thruster are completely independent of each other except for sharing a common facility ground, the most likely explanation for this synchronization is cross talk through the plasma plume. Since the interval between data points is approximately 25 seconds, variations in discharge current shown in Figure 6 do not depict highfrequency oscillations. Rather, the data represent quasisteady state changes in the thruster operating conditions.

The most likely explanation for the synchronization of the discharge currents lies in the concept of plasma resistivity. Although the resistivity of a fully ionized plasma is generally considered to be independent of the electron density, this is not the case for a partially ionized plasma such as the one found in the plume of a Hall thruster. ${ }^{11}$ In this case, the resistivity is inversely proportional to the electron density. When the discharge current of one thruster increases, for whatever reason, it is reasonable to assume that the plasma density increases slightly since one would not expect the ion or electron velocity to increase without a change in discharge voltage. When the plasma density increases, the resistivity drops slightly, and the adjacent thrusters respond with an increased discharge current. High-speed measurements of the discharge currents are planned in order to explore this phenomenon further.

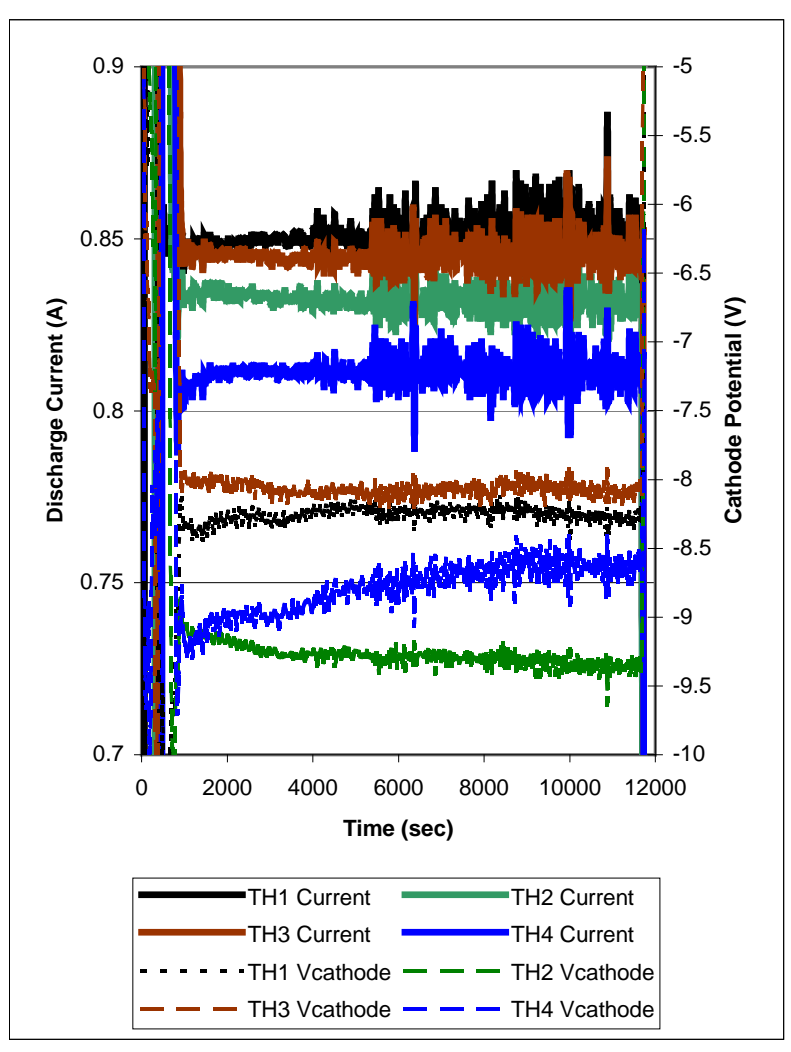

Figure 5: Variation of discharge current and cathode potential.

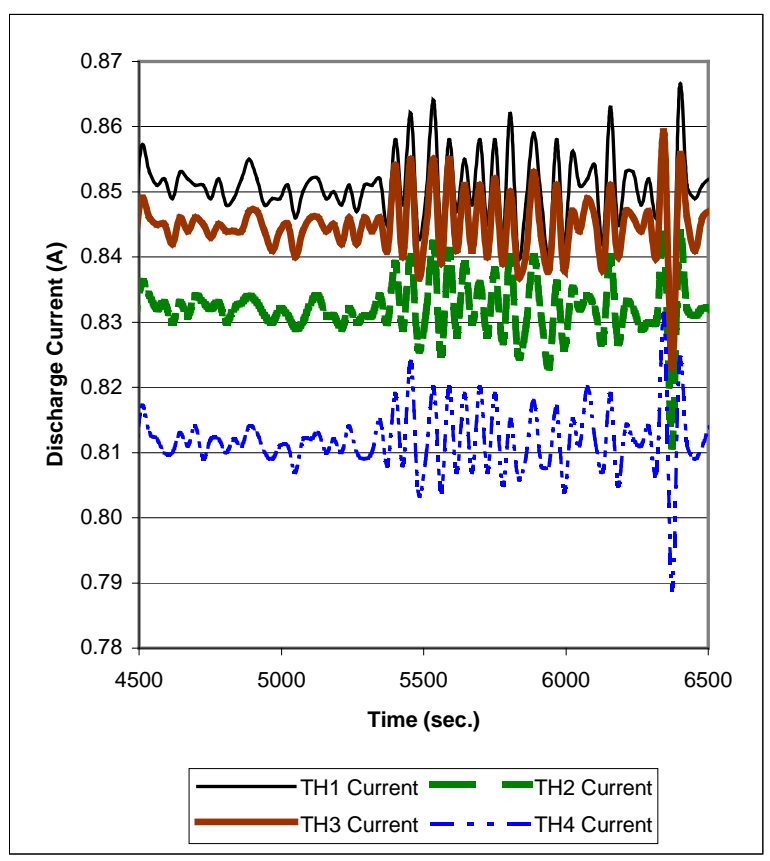

Figure 6: Close-up of discharge current fluctuations. Note the synchronization of the variations. 


\section{Plume Properties}

Data from the triple probe were recorded at 3 $\mathrm{cm}$ intervals over a $30 \mathrm{~cm} \times 30 \mathrm{~cm}$ area in the plane perpendicular to the thrust axis and at axial locations ranging from 5 to $35 \mathrm{~cm}$ downstream of the exit plane $(\mathrm{Z}=0-300 \mathrm{~mm}$ in the stated coordinate system). Due to a failure in the positioning system, the data taken for the $\mathrm{Z}=300 \mathrm{~mm}$ plane are unreliable. The original plan for this experiment was to first take data at $3 \mathrm{~cm}$ intervals and then take more closely spaced data points in areas of interest. Unfortunately, repeated failures of the stepper motors used in the positioning system, as well as an electrical short that occurred in one of the cables leading to the triple probe after the $3 \mathrm{~cm}$ data were taken, made this impossible.

Figures 7-9 show the electron number density profiles in the planes perpendicular to the thrust vector at locations 80,200, and $320 \mathrm{~mm}$ downstream of the exit plane, respectively. In all of the figures that follow, positions are reported in units of millimeters. The plasma density ranged from approximately $1 \times 10^{18}$ $\mathrm{m}^{-3}$ near the exit plane to about $5 \times 10^{15} \mathrm{~m}^{-3}$ in the farfield regions. These figures show the evolution of the plasma plumes as they proceed downstream and merge into a single plume structure from the four distinct plumes seen in the upstream region. Notice the four distinct plumes shown in Figure 7 and the dip in electron density near the center of the cluster. Further downstream, in Figure 8, the dip in density near the center of the cluster is much less pronounced, and in Figure 9 the plumes have merged to such a degree that the existence of four distinct plumes is no longer obvious. In Figure 7, the mesh of points where data were taken is overlaid on the plot to show the poor spatial resolution of the current measurements. Data were collected at each of the vertices shown. Future experiments with a much smaller interval between data points are planned.

Figures 10-12 show electron number density profiles in front of thrusters 1 and 2, along the central plane of the cluster, and in front of thrusters 3 and 4 , respectively. These planes correspond to $\mathrm{X}=-60,0$, and $60 \mathrm{~mm}$. The planes in front of the thruster pairs are within $3 \mathrm{~mm}$ of the thruster centerline in both cases. Note the well-defined jet structure shown in Figures 10 and 12. The apparent jaggedness of the plume is due to the large distance between data points. As one would expect, Figure 11 shows lower densities along the midplane of the cluster compared to the high densities measured along the thruster centerlines. Note that the reference scale differs among the plots of number density shown in Figures 7-12. The wide range of plasma parameters displayed makes this scale change necessary to show the structure of the plasma plumes.

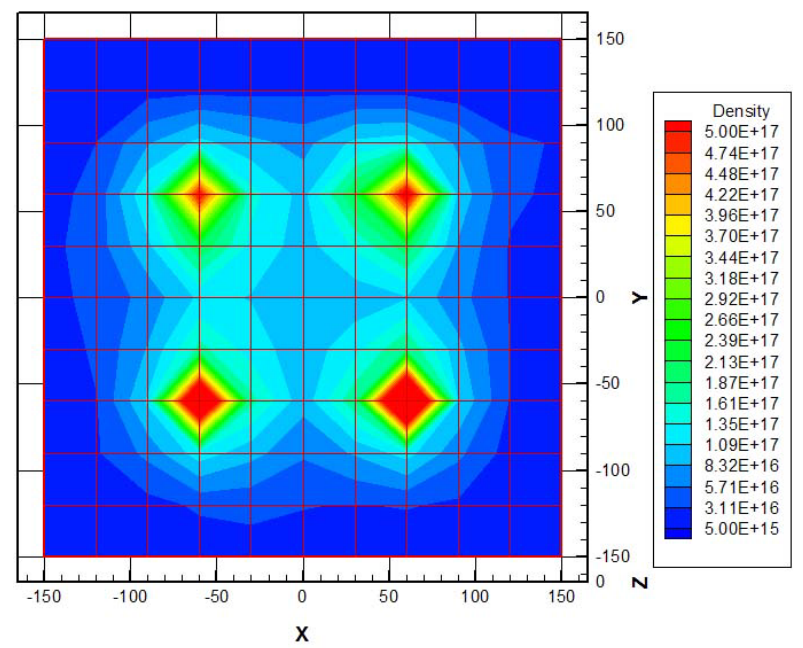

Figure 7: Electron number density $\left(\mathrm{m}^{-3}\right) 80 \mathrm{~mm}$ downstream. Note the four distinct plumes and high density along thruster centerlines.

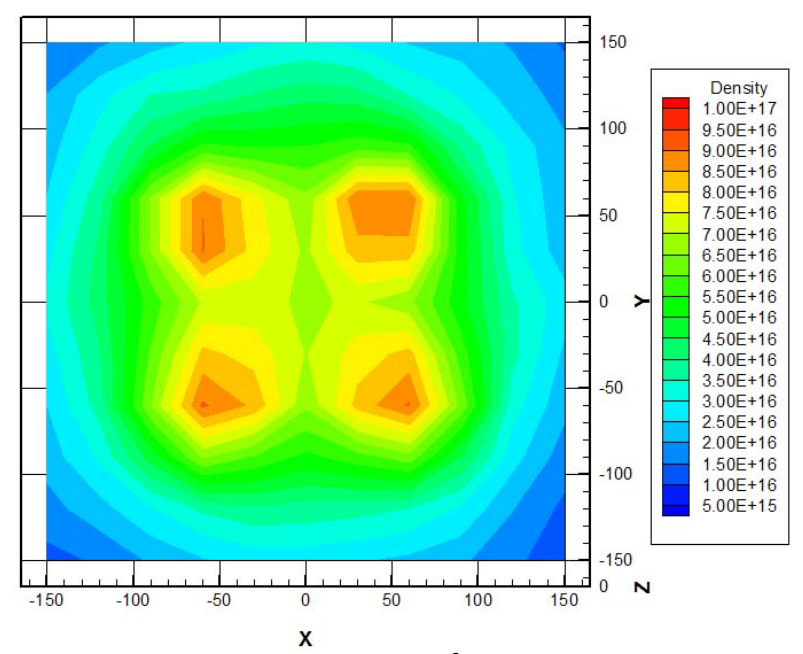

Figure 8: Electron density $\left(\mathrm{m}^{-3}\right) 200 \mathrm{~mm}$ downstream. Note the scale change from Figure 7.

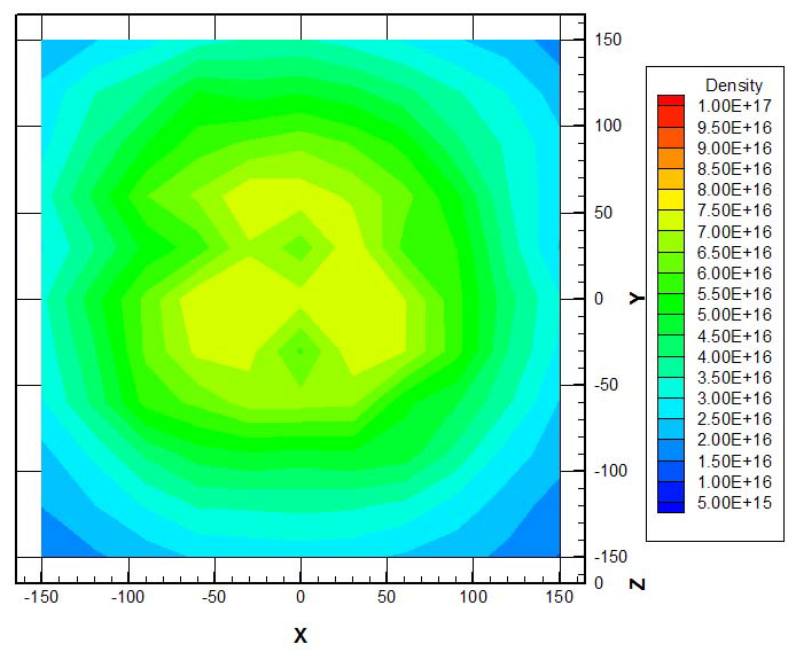

Figure 9: Electron density $\left(\mathrm{m}^{-3}\right) 320 \mathrm{~mm}$ downstream. 


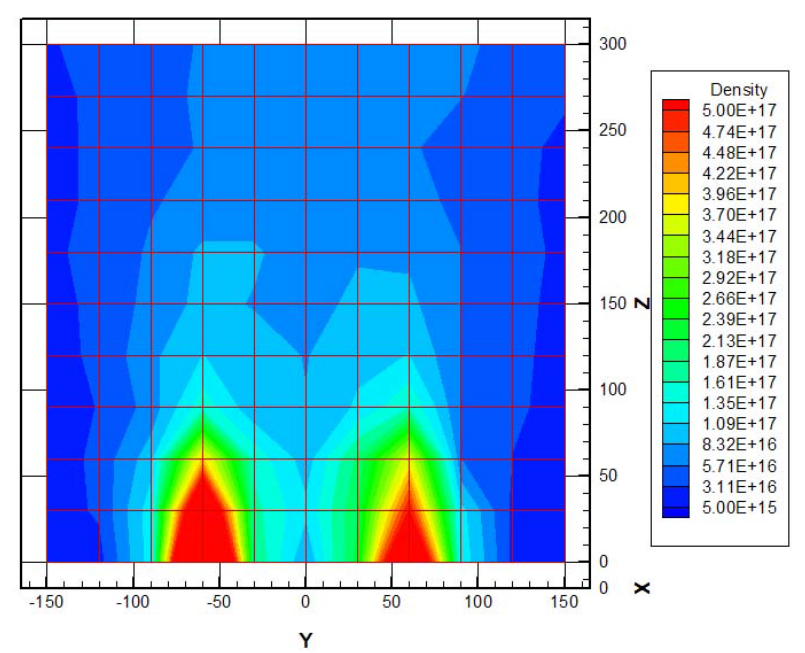

Figure 10: Electron number density $\left(\mathrm{m}^{-3}\right)$ along the centerlines of thrusters 1 and 2 .

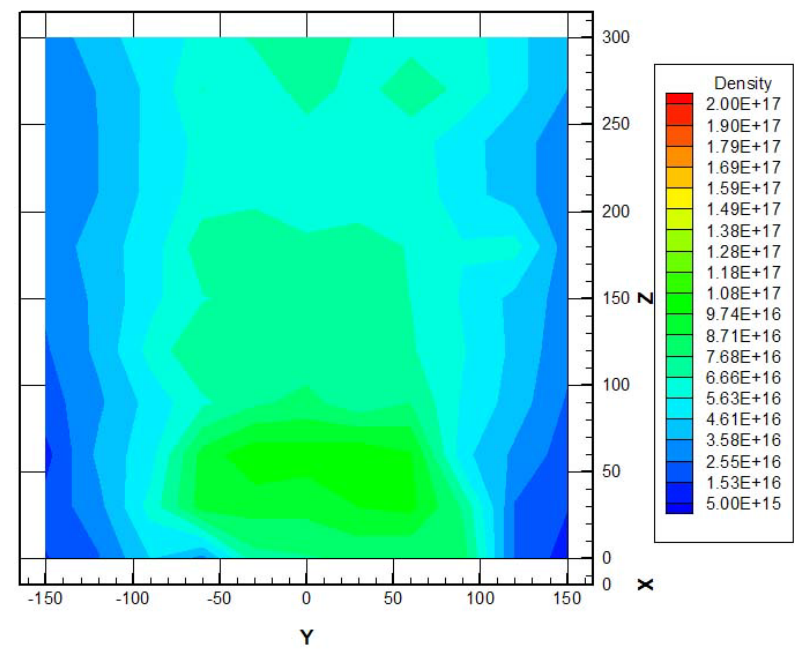

Figure 11: Electron density $\left(\mathrm{m}^{-3}\right)$ on cluster centerline. Note the scale change from Figures $10 \& 12$.

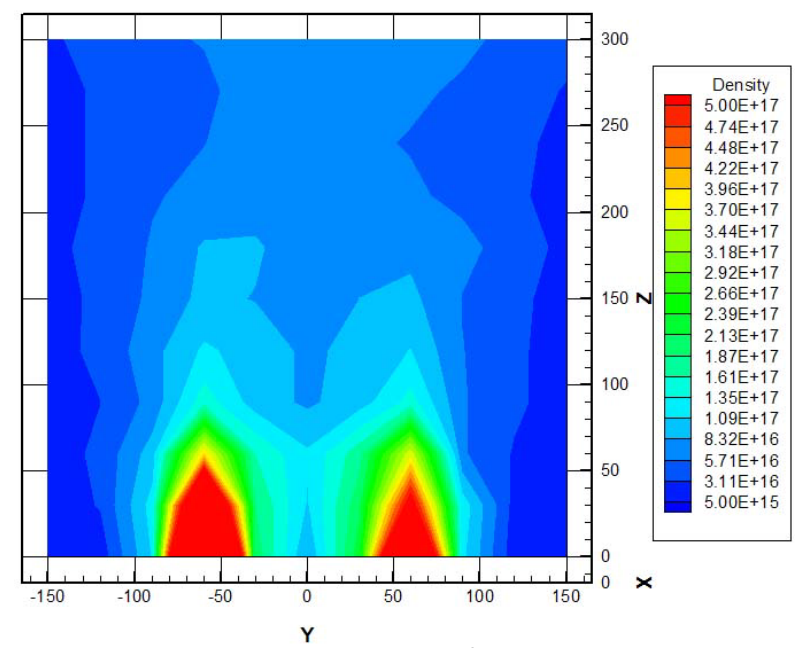

Figure 12: Electron density $\left(\mathrm{m}^{-3}\right)$ along the centerlines of thrusters 3 and 4 .
The measured electron temperature along various planes in the plume of the cluster is displayed in Figures 13-18. Figures 13-15 are cross sections taken perpendicular to the thrust vector while Figures 16-18 were taken perpendicular to the exit plane. The locations of these measurements correspond to those shown in Figures 7-12. There are several very high electron temperatures reported, particularly in Figure 13, but also at isolated points in Figures 16-18. These isolated points are inconsistent with the overall structure of the data and seem to occur in areas of extremely low density near the boundaries of the sampled volume. Since the Debye length, and hence the thickness of the plasma sheath surrounding each electrode, is inversely proportional to the square root of the plasma density, the thin sheath approximation (Eqn. 2 and 4) is not justified in regions of very low density. For this reason, the unusually high values of electron temperature shown near the top and bottom edges of Figure 13 and near the corners of Figures 16-18 are not considered reliable. This issue is not a factor near the center of each figure and farther downstream where the plume divergence insures that the plasma density is high enough to satisfy the requirements of probe theory presented previously.

The electron temperature near the thruster exit planes was measured at $1-2 \mathrm{eV}$. It then increases to 4-5 $\mathrm{eV}$ approximately $14 \mathrm{~cm}$ downstream before falling off to $2-3 \mathrm{eV}$ in the far-field regions. Figures 14 and 15 show that the measured electron temperature is slightly higher near the centerline of each thruster than it is elsewhere in the plumes. The fact that the increase in electron temperature occurs downstream of all four thrusters, as indicated in Figures 16 and 18, seems to indicate that it is a real phenomenon and not the result of bad data points. Figure 17 shows that the increase in temperature does not occur along the midplane of the cluster. This leads one to believe that the effect is associated with each of the individual thrusters and is not a consequence of clustering. The suspected explanation for this increase in electron temperature involves the two-stream instability and the possible formation of collisionless shock waves, which will be discussed in subsequent sections of this paper. Hot electrons coming directly from each cathode have also been suggested as a possible cause of the regions of high electron temperature, however if this were the case there would be no reason to expect that the hot spots would occur only along the thruster centerlines. Therefore, cathode phenomena are not believed to be responsible for the regions of high electron temperature. 


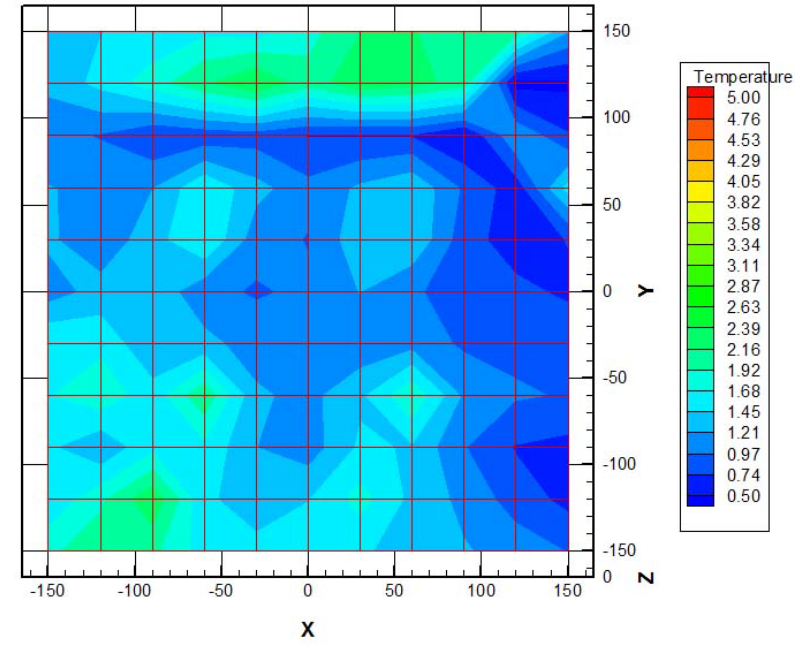

Figure 13: Electron temperature (eV) $80 \mathrm{~mm}$ downstream.

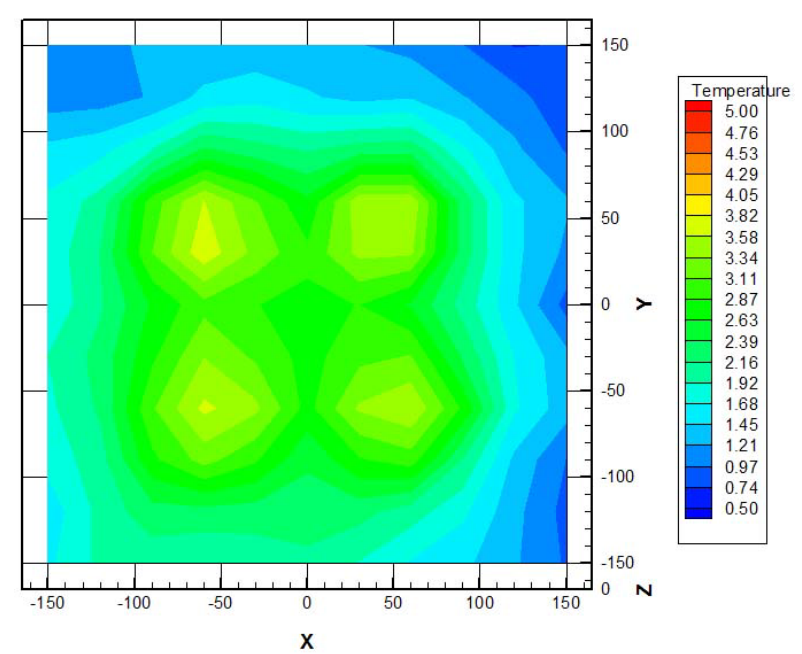

Figure 14: Electron temperature (eV) $200 \mathrm{~mm}$ downstream.

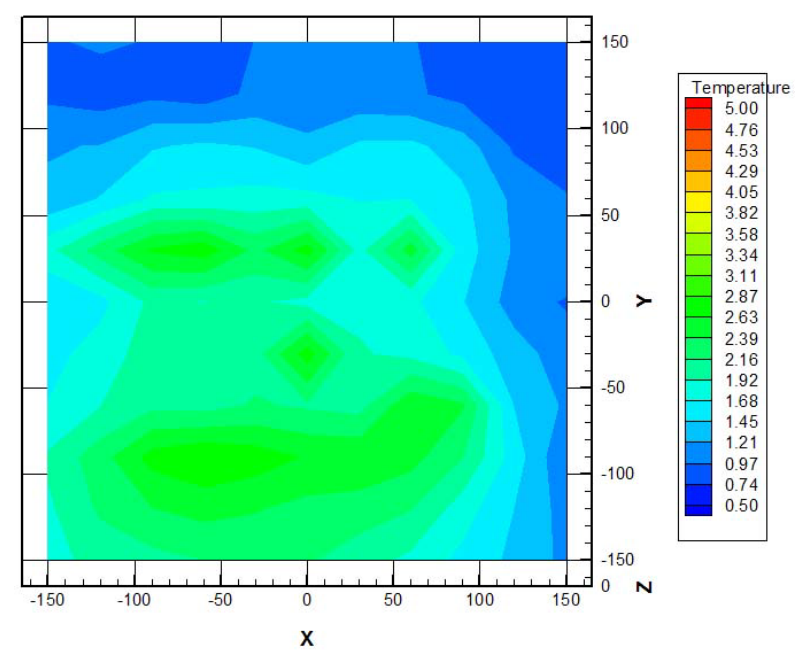

Figure 15: Electron temperature (eV) $320 \mathrm{~mm}$ downstream.

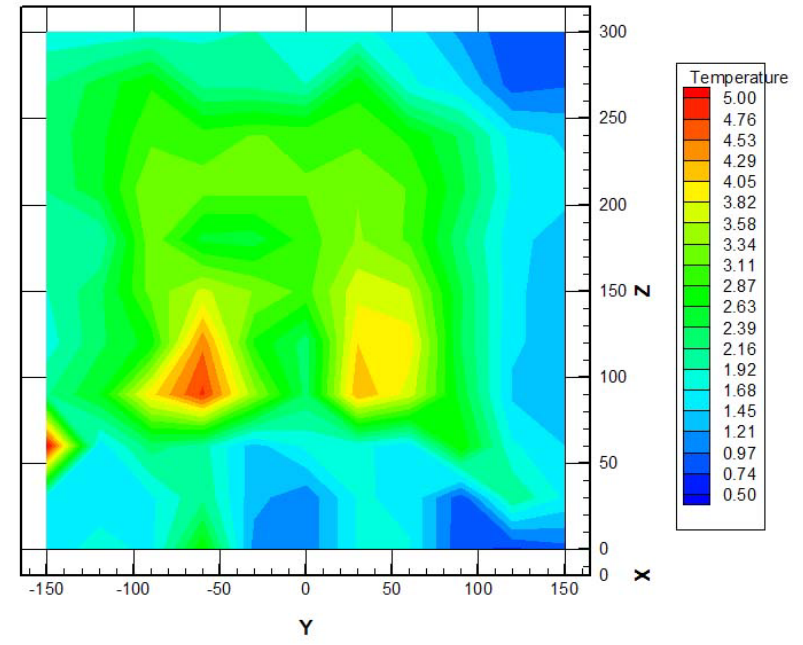

Figure 16: Electron temperature $(\mathrm{eV})$ at $X=-60 \mathrm{~mm}$.

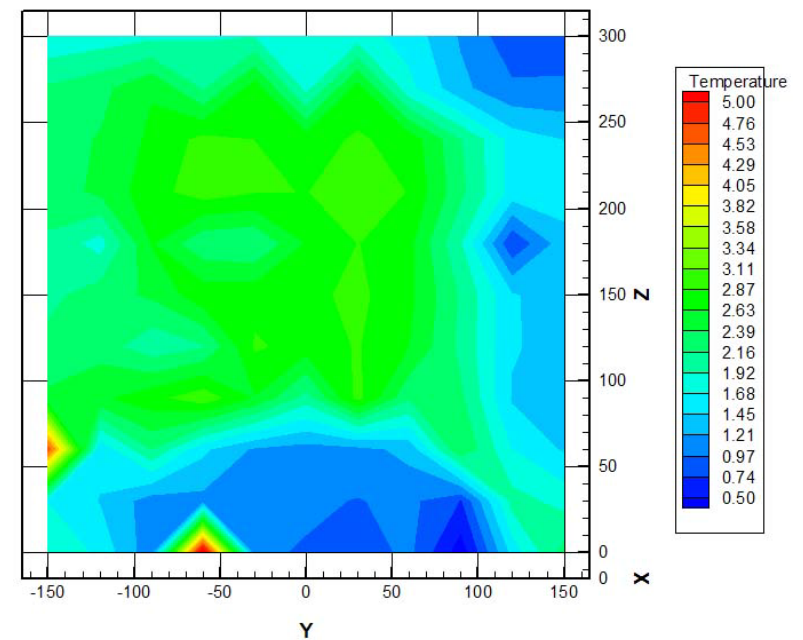

Figure 17: Electron temperature $(\mathrm{eV})$ at $\mathrm{X}=0 \mathrm{~mm}$.

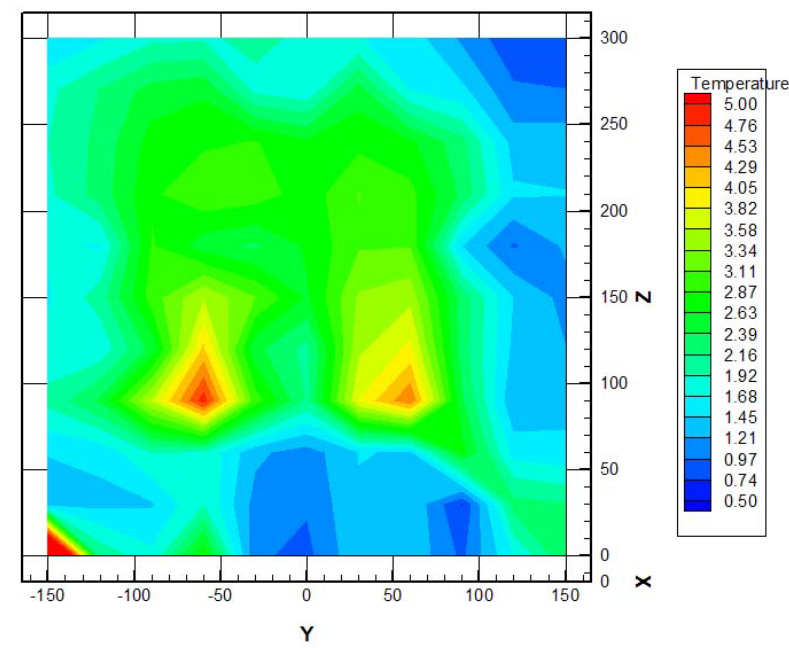

Figure 18: Electron temperature $(\mathrm{eV})$ at $X=60 \mathrm{~mm}$. 
Plasma potential profiles are shown in Figures 19-24. The presented locations are the same as those shown for the number density and electron temperature. The potential varies between 2 and 25 volts for most of the sampled volume. Regions of high plasma potential generally correspond to regions of high electron temperature as expected considering the relation between the two properties shown in Eqn. 8. The structure of Figures 19 and 20 show that the plasma potential is higher along the centerline of each thruster than it is in the surrounding plasma. In Figure 21, the plasma potential changes very little across the entire plume. This is consistent with the structure shown in Figures 9 and 15 for the electron density and temperature, respectively. Figures 22-24 show marked increases in plasma potential along the centerline of each thruster on the order of 10 volts with respect to the surrounding plasma. These areas of high plasma potential are analogous to the hot spots shown in the electron temperature data. Like the electron temperature plots, there are several questionable data points in the plasma potential contours $80 \mathrm{~mm}$ downstream of the exit plane, however the other plots show a reasonable structure. The explanation for the erroneous data points is the same as the one discussed previously for the electron temperature. Since the electron temperature and plasma potential are related through Eqn. 8, it is not surprising that errors in the measurements at several data points are reflected in both data sets.

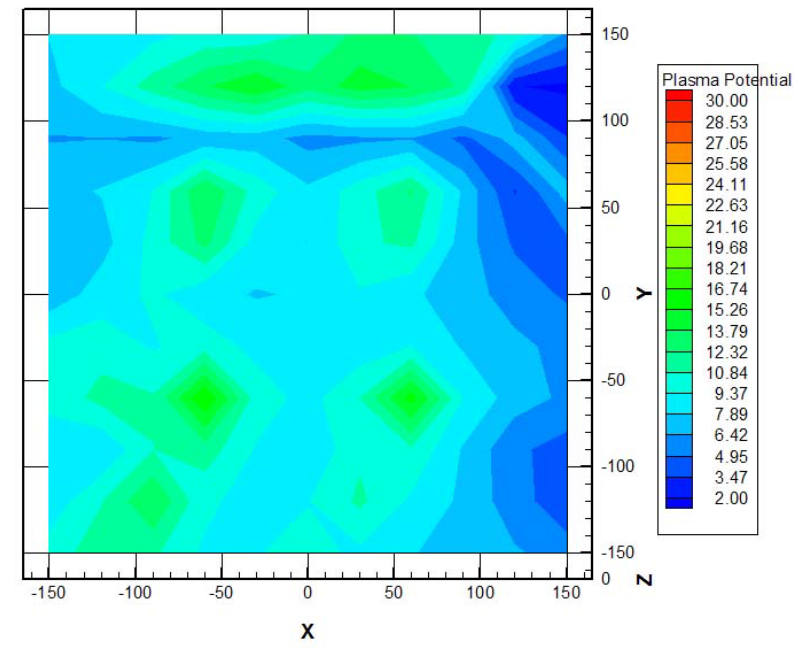

Figure 19: Plasma potential (volts) $80 \mathrm{~mm}$ downstream.

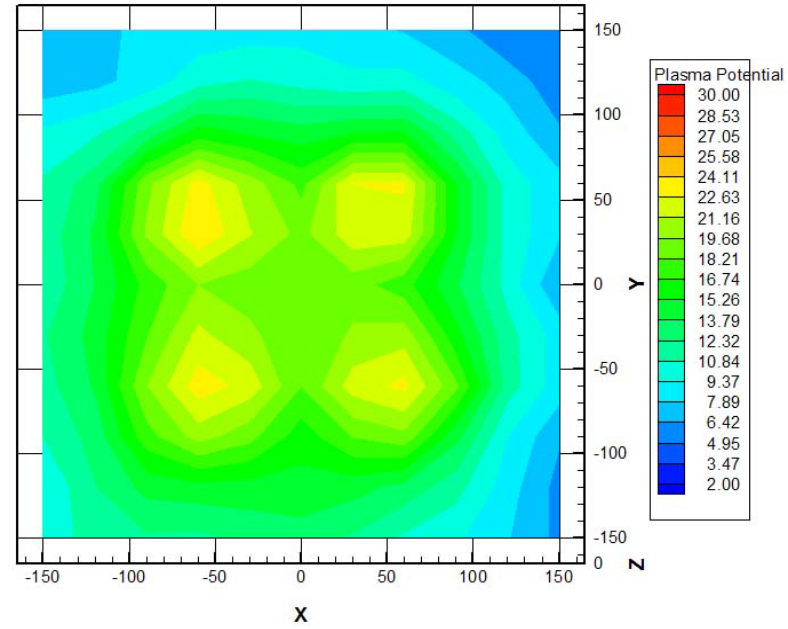

Figure 20: Plasma potential (volts) $200 \mathrm{~mm}$ downstream.

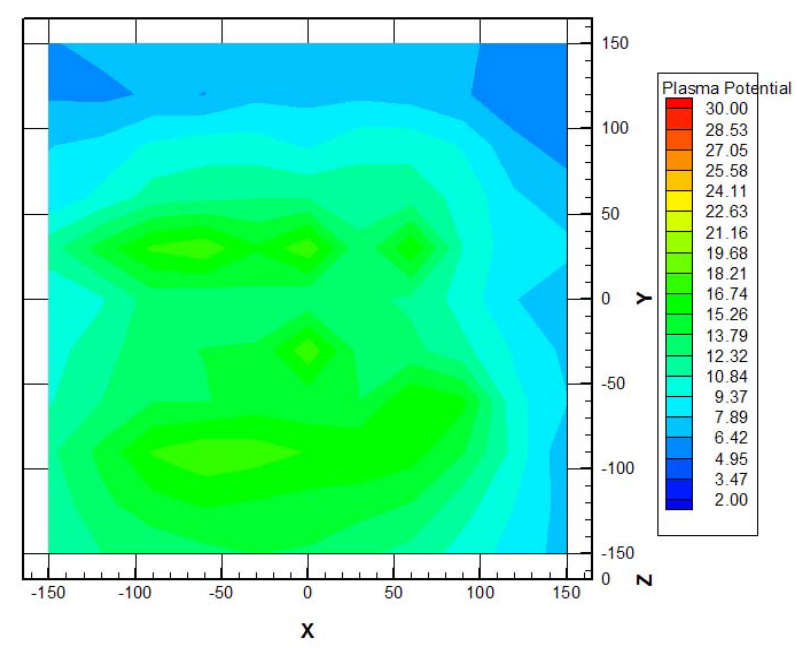

Figure 21: Plasma potential (volts) $320 \mathrm{~mm}$ downstream.

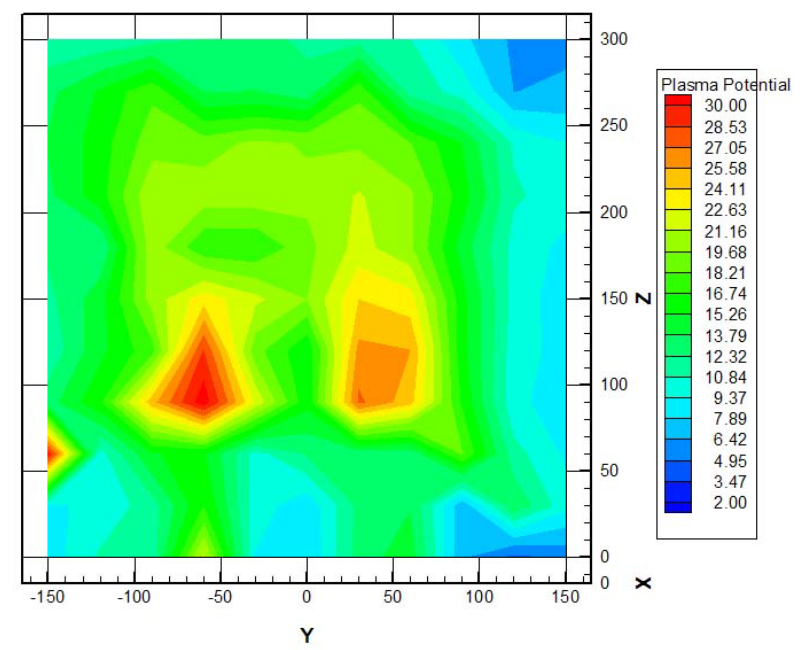

Figure 22: Plasma potential (volts) in front of thrusters 1 and 2 . 


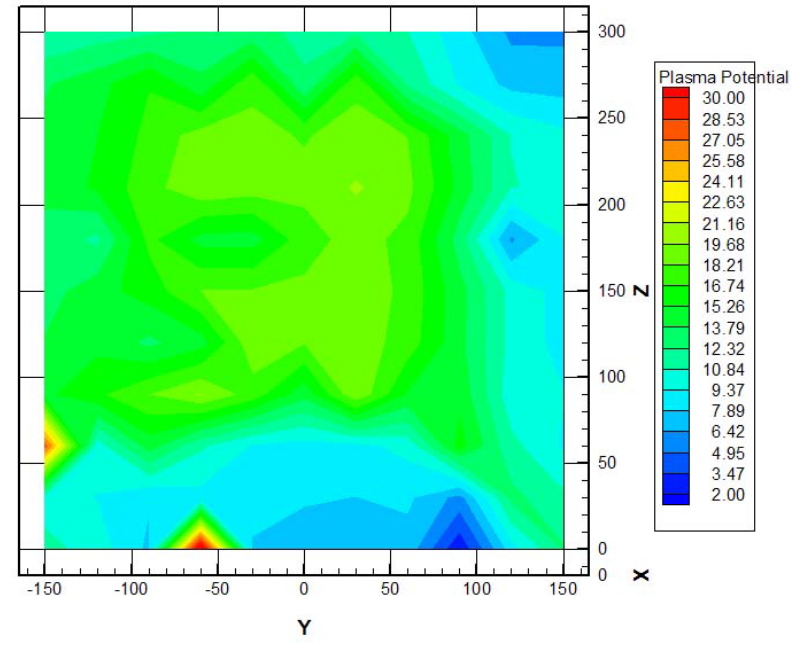

Figure 23: Plasma potential (volts) on cluster centerline.

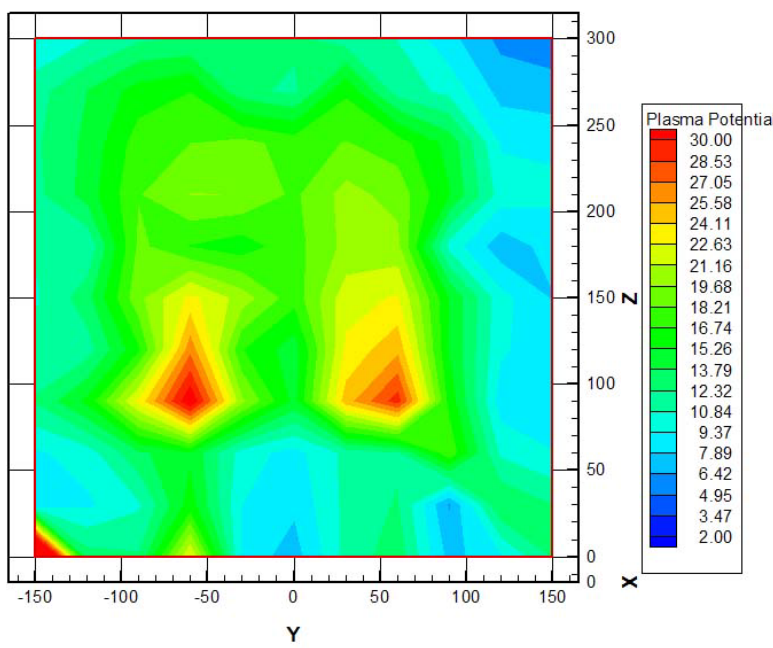

Figure 24: Plasma potential (volts) in front of thrusters 3 and 4.

\section{$\underline{\text { Discussion }}$}

The electron temperature and plasma potential measurements presented in the previous section show very interesting features along the centerline of each thruster that seem to coincide with the bright cores visible in Figure 2. The bright cores occur downstream of each thruster in the cluster and have been observed in many other Hall thruster plumes, therefore it is reasonable to consider the properties of an individual thruster when attempting to determine the causes of the high electron temperature and plasma potential presented previously. Although the following discussion is somewhat hypothetical in nature, it offers a physical explanation for the observed phenomena.

\section{Two-stream Instability}

In an annular thruster, such as the BHT-200$\mathrm{X} 3$, a portion of the ion beam converges along the centerline of the thruster, as sketched in Figure 25. This creates a situation where the ion beam from one part of the thruster passes through its mirror image as the two streams cross the centerline. There are several instabilities that can be excited in a situation like this, but before discussing each of them it is helpful to consider some of the key plasma parameters in the plume so that these estimates can be used to determine which instabilities are important. Table 2 presents several important parameters for a plasma with density, $\mathrm{n}_{\mathrm{e}}=1 \times 10^{17} \mathrm{~m}^{-3}$, electron temperature, $\mathrm{T}_{\mathrm{e}}=2 \mathrm{eV}$, and drift velocity, $V_{\text {drift }}=17,000 \mathrm{~m} / \mathrm{s}$, which are approximate values taken from the presented triple probe data and an estimate of the ion drift velocity based on the assumption that the ions are accelerated through a 200 volt potential drop. The ion temperature is taken to be $0.5 \mathrm{eV}$, consistent with Ref. 12 and 13. The magnetic field, $\mathrm{B}$, is assumed to be 20 gauss, which is less than $10 \%$ of the value inside the discharge channel. These numbers are meant only to show the scale of various parameters and are not necessarily quantitatively accurate. All quantities were calculated for xenon using the approximate formulas in Ref. 14.

\begin{tabular}{|c|c|}
\hline Parameter & $\begin{array}{c}\text { Approximate } \\
\text { Value }\end{array}$ \\
\hline Electron thermal speed, $\mathrm{v}_{\mathrm{e}},(\mathrm{m} / \mathrm{s})$ & $5.9 \times 10^{5}$ \\
\hline Ion thermal speed, $\mathrm{v}_{\mathrm{i}},(\mathrm{m} / \mathrm{s})$ & 600 \\
\hline Ion acoustic speed, $\mathrm{C}_{\mathrm{s}},(\mathrm{m} / \mathrm{s})$ & 1,500 \\
\hline Alfven speed, $\mathrm{C}_{\mathrm{A}},(\mathrm{m} / \mathrm{s})$ & 12,000 \\
\hline Beta, $\beta=8 \pi \mathrm{nk}_{\mathrm{b}} \mathrm{T}_{\mathrm{e}} / \mathrm{B}^{2}$ & 0.02 \\
\hline $\begin{array}{c}\text { Electron plasma frequency, } \omega_{\mathrm{pe}}, \\
(\mathrm{rad} / \mathrm{s})\end{array}$ & $1.8 \times 10^{10}$ \\
\hline $\begin{array}{c}\text { Electron cyclotron frequency, } \\
\omega_{\text {ce }},(\mathrm{rad} / \mathrm{s})\end{array}$ & $3.5 \times 10^{8}$ \\
\hline Electron Larmor radius $(\mathrm{mm})$ & 1.7 \\
\hline
\end{tabular}

Table 2: Estimated plasma properties in the near-field cluster plume. 


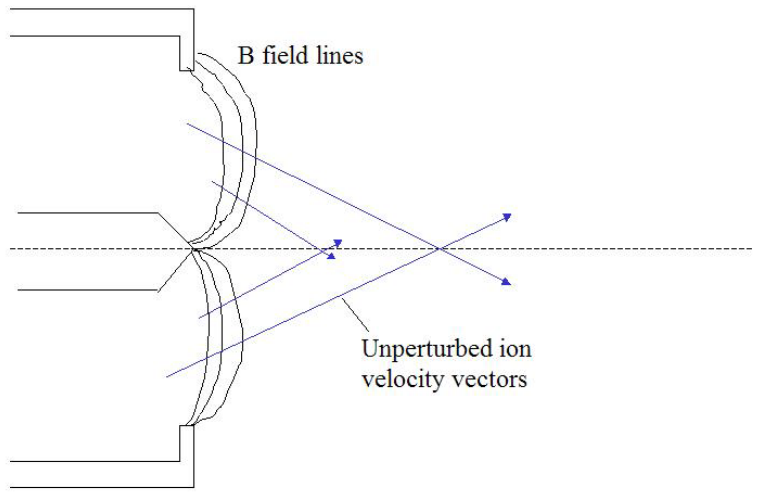

Figure 25: Convergent ion trajectories that lead to the onset of the two-stream instability.

There are several instabilities that should be considered for a convergent plasma beam. The first is the electron-electron (e-e) two-stream instability. Since the electron thermal speed in the plume is two orders of magnitude higher than the relative drift speed between the two beams, the interactions between the electrons are stable. ${ }^{15}$

The second instability to consider is the electron-ion (e-i) two-stream instability, which can occur between the ions of one beam and the electrons of another. This mechanism requires the ion temperature to be approximately equal to the electron temperature and the drift velocity to be greater than the electron thermal velocity. ${ }^{16}$ Since the drift speed is much less than the electron thermal speed in the current situation, this mode can immediately be eliminated as a possibility.

Another instability of interest is the ion acoustic instability, which occurs when electrons drift relative to ions and $\mathrm{T}_{\mathrm{e}} \gg \mathrm{T}_{\mathrm{i}}$. Strictly speaking, this mode may be slightly unstable in the current situation, however it is doubtful that this could significantly affect the plasma parameters. It can be shown by quasilinear theory that this instability usually serves only to flatten the electron energy distribution in the narrow region of velocity space where wave interactions can occur. ${ }^{11}$ It generally does not affect the ion energy distribution and the effect on the electrons is limited to decreasing the slope of the distribution function, $\mathrm{df}_{\mathrm{e}} / \mathrm{dv}$, in a narrow region of velocity space such that the large scale structure is not altered significantly. In this case, the ion acoustic instability would not be expected to significantly alter macroscopic quantities such as electron number density and electron temperature.
The final, and most important, mode to consider is the ion ion (i-i) two-stream instability. It is tempting to declare this mode stable since linear theory shows that the drift speed must be less than the ion acoustic speed for momentum transfer to occur between the ions in an unmagnetized plasma. ${ }^{16-18}$ This requirement stems from the premise that the wavelength of the disturbance must be shorter than the Debye length such that electrons cannot effectively neutralize disturbances caused by ion bunching. It has been shown, however, that the presence of even a weak magnetic field significantly alters the range of instability. ${ }^{18}$ Papadopoulos et al., have considered the case where two ion beams flow into each other across a magnetic field. The magnetic field strength was such that electrons were magnetized, but the ion trajectories were unaffected by the magnetic field. This work showed that counterstreaming ion beams in the presence of a magnetic field are unstable even for very small wavenumbers, $\mathrm{k}$, which are defined by Eqn. 9 where $\lambda$ is the wavelength of the oscillations. This leads to the conclusion that the $\mathrm{i}-\mathrm{i}$ two-stream instability can grow even for drift velocities much larger than the ion acoustic speed when a weak magnetic field is considered.

$$
k \equiv \frac{2 \pi}{\lambda}
$$

The $\mathrm{i}-\mathrm{i}$ mode becomes even more unstable when one abandons the one-dimensional approximation and considers convergent beams in cylindrical geometry. ${ }^{19}$ In this case, the instability shows no lower limit on the unstable wavenumbers and the growth rate is larger than for the corresponding planar system. ${ }^{19}$ Since this cylindrical geometry more accurately reflects the physical situation downstream of a Hall thruster, it is clear that the ion-ion two-stream instability is unstable in the near-field plume.

\section{Collisionless Shocks}

There are at least three reasons to suspect that collisionless shocks may occur in the plume of a Hall thruster. First, ion acoustic shocks have been proposed as an explanation for the well defined boundaries of the bright core seen along the centerline of each thruster in Figure 2. ${ }^{9} \quad$ Second, laser induced fluorescence (LIF) measurements obtained by Smith et al. have shown a significant population of ions along the centerline of a Hall thruster having nearly zero radial velocity. ${ }^{20}$ Since collisions are too rare to deflect a significant population of the plasma into this region of velocity space and there is no way for an ion to reach the centerline with zero radial velocity unless it is deflected somehow, it is reasonable to believe that the deflection mechanism could be a collisionless shock wave. Finally, it has 
been well established, both analytically and experimentally, that the ion-ion two-stream instability discussed above can cause the dissipation necessary to form a collisionless shock. ${ }^{16,21-23}$

The theory of collisionless shock waves is a very deep and complex field, a thorough treatment of which is beyond the scope of this paper. A brief review of several key aspects, however, is necessary to determine the applicability of collisionless shocks to the plume of a Hall thruster. Due to the lack of attention in the literature to shock waves in cylindrical coordinates, as well as their inherently greater complexity, it is convenient to consider only one-dimensional shocks. While this approach cannot be expected to give quantitatively accurate results, it does give useful insight into shock physics and the changes in plasma parameters across a shock.

Although it seems counterintuitive to consider the effect of the relatively weak magnetic field found in the plume of the Hall thruster, there is reason to believe that a rigorous discussion of collisionless shock waves in the thruster plume should include it. Tidman and Krall have shown that magnetosonic waves are dominant over ion acoustic waves in a low beta plasma such as the one described by Table $2 .^{23}$ Further evidence of the need to include the effect of the magnetic field is found by comparing the linear dispersion relations for both magnetosonic and ion acoustic waves, which are given in Eqn. 10 and 11, respectively, where $\omega$ is the frequency, $k$ is the wavenumber, and $\mathrm{c}$ is the speed of light. ${ }^{24}$ As these relations show, the magnetosonic wave speed depends on both the ion acoustic and Alfven speeds, which are given in Eqn. 12 and 13, respectively. Since the Alfven speed has been estimated to be several times larger than the acoustic speed, the magnetosonic wave, which depends on the magnetic field strength, is expected to dominate. Fortunately, because the magnetosonic dispersion relation simplifies to the ion acoustic relation when the magnetic field strength approaches zero, it is possible to consider the magnetosonic shock without losing any information about the ion acoustic shock that forms in this limit.

$$
\begin{gathered}
\frac{\omega^{2}}{k^{2}}=c^{2} \frac{C_{s}^{2}+C_{A}^{2}}{c^{2}+C_{A}^{2}} \\
\frac{\omega^{2}}{k^{2}}=C_{s}^{2}
\end{gathered}
$$

$$
\begin{gathered}
C_{s}=\sqrt{\left(\frac{k_{B} T_{e}}{m_{i}}\right)} \\
C_{A}=\frac{B}{\sqrt{4 \pi m_{i} n}}
\end{gathered}
$$

When the magnetic field is considered, there are three speeds that become important, the slow magnetosonic, ion acoustic, and fast magnetosonic speed. ${ }^{25}$ A collisionless shock can be based on either the fast or the slow wave depending on the geometry of the problem. The factor determining which wave speed a shock will be based on is the value of the so called intermediate speed, which is defined as $C_{A} \cos (\theta)$, where $\theta$ is the angle between the upstream magnetic field and the wave propagation vector as shown in Figure 26. For the more common fast magnetosonic shock, both the upstream and downstream flow velocities must be greater than the intermediate speed. ${ }^{25}$ The opposite is true for the slow shock. The main difference between the two types is that the magnetic field increases across the fast shock and decreases across the slow shock. For the quasiperpendicular shock, in which $\theta$ approaches $90^{\circ}$, the dominant wave mode is the fast one since the intermediate velocity goes to zero. In the present case, $\theta$ is believed to approach $90^{\circ}$ based on the assumed magnetic field profile and the observed location of the bright shock cone. Although a quasiperpendicular, fast shock will be assumed throughout the rest of this discussion, the possibility of oblique, slow shocks cannot be totally eliminated without accurate measurements of the magnetic field strength and direction in the vicinity of the shock.

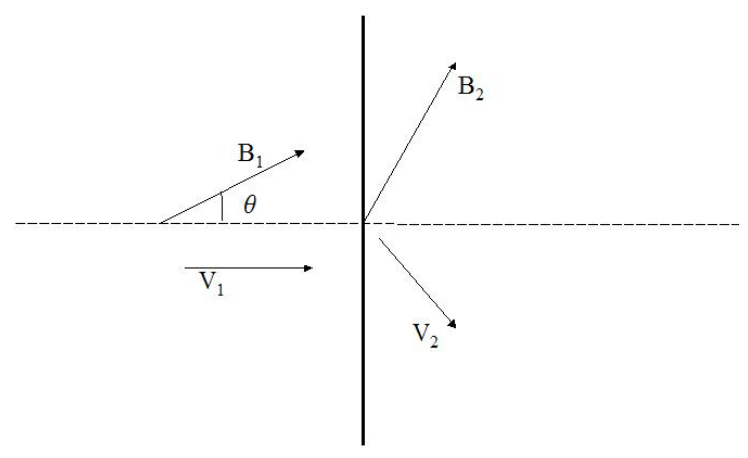

Figure 26: Shock nomenclature.

Having decided that any shock present in the system is likely to be a perpendicular magnetosonic shock, it would be convenient to predict the properties 
downstream of the shock using the Rankine-Hugoniot relations given in Ref. 23. Although these relations are mathematically tractable, their utility is limited because they require knowledge of the magnetic energy, which may be significant in the very low beta plasmas being considered. In fact, the Rankine-Hugoniot equations are so sensitive to the magnetic field strength that attempts to use them to predict plasma properties downstream of a shock are futile without much better estimates for the magnetic field strength than those reflected in Table 2. For this reason, a rigorous application of these relations will be postponed until after measurements of the magnetic field have been obtained. Additionally, the usefulness of the RankineHugoniot relations is diminished by their inability to determine the partition between the ion and electron temperatures, and their assumption of Maxwellian velocity distributions on both sides of the shock. While this may be a fair assumption upstream, there is no reason to believe that the downstream flow should be in equilibrium considering the collisionless nature of the plasma. $^{23}$ As a side note, this brings up an interesting point concerning the diagnostic used in this experiment. Since the derivation of the triple probe equations assumed a Maxwellian plasma, it is conceivable that any measurements taken downstream of a shock may be quantitatively inaccurate.

A useful alternative to the Rankine-Hugoniot relations is a very simple model based solely on geometric arguments and observations of measured collisionless shocks. This method cannot be expected to produce quantitatively accurate results, because it neglects effects such as the thermal spread of the ion velocities on both sides of the shock and the changes in magnetic field strength. It can, however, be used to answer several questions about the shock. In particular, this model explains an apparent discrepancy in the reported data, which shows an increase in electron temperature without a clear change in plasma density. The geometry and nomenclature used for this simple model are given in Figure 27.

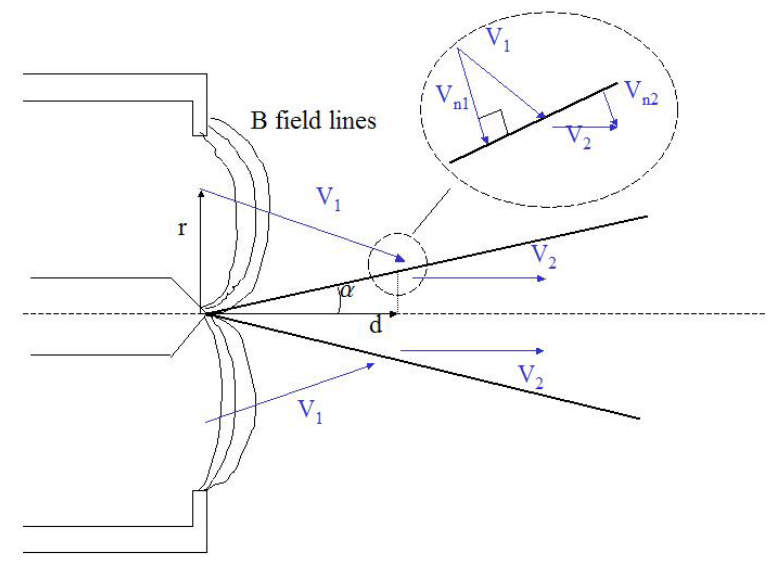

Figure 27: Simple shock model.

The model illustrated in Figure 27 gives a crude estimate of the changes in plasma properties as a function of the shock divergence angle, $\alpha$, the radial location in the exit plane where a sample ion is "born", $\mathrm{r}$, and the downstream distance where that ion intersects the shock surface, d. The model depends on several limiting assumptions and geometric arguments, namely:

i.) The shock only affects the normal component of velocity such that, $\mathrm{V}_{\mathrm{t} 1}=\mathrm{V}_{\mathrm{t} 2}$, $\mathrm{V}_{\mathrm{n} 1}>\mathrm{V}_{\mathrm{n} 2}$.

ii.) The shock must turn the flow so that downstream of the discontinuity the flow is directed parallel to the thruster centerline.

iii.) Ions follow straight lines from the exit plane to the shock interface and are not subjected to collisions or external electric and magnetic fields.

iv.) At all points for which this model is applied, $r>d \sin (\alpha)$.

The assumptions listed above have several weaknesses. In particular, assumption ii overstates the change in plasma flow because a shock needs only rearrange the velocity distribution such that the downstream distribution is stable. It does not necessarily turn all ions parallel to the centerline. Assumption iii is not strictly accurate for a Hall thruster plume since a significant portion of the ion acceleration has been shown to occur downstream of the exit plane. The cumulative effect of these weaknesses is to limit the range of parameters over which the model gives reasonable results. Specifically, this simple model is only reasonable for $\mathrm{d} / \mathrm{r} \quad 3$ such that the change in ion direction is not too large. Assumption iii limits the estimates to situations where the change in energy associated with the magnetic field is small compared to the change in kinetic and thermal energy across the 
shock. Using the assumptions mentioned above, it is possible to write the normal component of upstream and downstream ion velocity as given by Eqn. 13 and 14. These can be used to calculate the change in number density and velocity across the shock as given by Eqn. 15 .

$$
\begin{gathered}
\frac{V_{n 1}}{V_{1}}=\frac{\cos (\alpha)}{\sqrt{\left(1-\frac{d}{r} \tan (\alpha)\right)^{2}+\left(\begin{array}{c}
d)^{2} \\
r
\end{array}\right)}} \\
\frac{V_{n 2}}{V_{1}}=\sin (\alpha)\left[\frac{\frac{d}{r}+\frac{d}{r} \tan ^{2}(\alpha)-\tan (\alpha)}{\sqrt{\left(1-\frac{d}{r} \tan ^{2}(\alpha)\right)^{2}+\left(\frac{d}{r}\right)^{2}}}\right] \\
\frac{n_{2}}{n_{1}}=\frac{V_{n 1}}{V_{n 2}}=\frac{\frac{d}{r}+\frac{d}{r} \tan ^{2}(\alpha)-\tan (\alpha)}{\Delta\left(\frac{k_{B}}{e}\right) \cong \frac{\zeta m_{i}}{2 e}\left(V_{n 1}^{2}-V_{n 2}^{2}\right)} \\
\Delta \phi \cong \frac{m i}{2 e}\left(V_{n 1}^{2}-V_{n 2}^{2}\right)
\end{gathered}
$$

The electrostatic potential jump is, in general, dependent upon the frame in which it is measured. ${ }^{26,27}$ For the relatively weak shocks considered here, however, the frame dependence can be ignored and the potential jump can be estimated as the change in ion kinetic energy as given by Eqn. 16. ${ }^{28,29}$ The electron temperature jump across a collisionless shock has been shown empirically to be $5-20 \%$ of the incident flow ram energy. ${ }^{30,31}$ Surprisingly, this relation seems to be only weakly dependent on the upstream parameters such as shock geometry and Mach number. ${ }^{30}$ An estimate for the jump in electron temperature is given by Eqn. 17, where $\zeta$ is an empirical coefficient representing the fraction of dissipated ion kinetic energy that is converted to electron thermal energy. For the calculations discussed here, $\zeta$ is assumed to be 0.20 , which is near the upper bound of the estimated range, since observations have shown that weak shocks, such as those considered here, tend to produce a larger degree of electron heating than strong shocks. ${ }^{30}$
Considering the discussion above, the increase in electron temperature without a pronounced increase in plasma density shown in Figures 10, 12, 16, and 18 can be explained as being caused by the large mass of a xenon ion. A small change in ion velocity causes an equally small change in number density, but a relatively large change in kinetic energy due to the large mass of the xenon ion. As Eqn. 15 and 17 demonstrate, the change in number density depends only on the velocity change across the discontinuity while the electron temperature also depends on the ion mass. Consequently, the change in electron temperature is quite noticeable because it is directly related to the kinetic energy lost by the ions, rather than the density change across the shock. For example, if one uses the crude model discussed above and considers a shock divergence half angle of $10^{\circ}$, as observed in Ref. 9, and an initial ion kinetic energy of 200 volts, the electron temperature change of roughly $2 \mathrm{eV}$ shown in Figures 16 and 18 corresponds to a density change of less than $40 \%$. Considering the $30-50 \%$ margin of error typical of Langmuir probes, the low spatial resolution of the current measurements, and the large gradients in plasma density present in the near-field, it is entirely possible that the density rise across the proposed shock has been overlooked. Future experiments with improved spatial resolution should clarify this issue. Figure 28 shows the electron temperature change as a function of the density jump across a shock with $\alpha=10^{\circ}$ for 200 volt ions using the simple model.

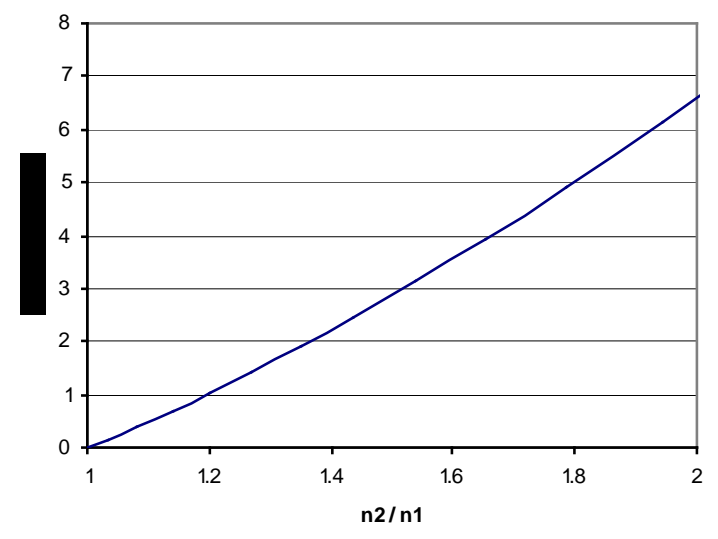

Figure 28: Electron temperature change versus density jump across a shock for 200 volt ions according to a simple geometric model.

\section{Implications for Clustering}

Considering that the ion-ion two-stream instability has been shown to be responsible for the formation of collisionless shocks in some situations, it is natural to ask whether this phenomenon could cause shocks in areas where the plumes of two thrusters 
intersect. While this possibility cannot be totally eliminated without additional measurements, it appears that formation of shocks at these intersections is unlikely. As was shown previously, intersecting ion beams with drift velocities much larger than the ion acoustic velocity are stable in the absence of a magnetic field for planar geometry. At the intersection of two plumes, the interface resembles planar geometry, rather than the convergent geometry along the centerline. Additionally, the magnetic field in the regions where plasma plumes could collide is expected to be much weaker than those in the near field of a single thruster. For these reasons, any instability induced by the intersecting beams can be expected to have a very low growth rate and hence be incapable of causing the dissipation necessary to induce a shock.

\section{$\underline{\text { Conclusions }}$}

Preliminary measurements of thruster operating conditions for a cluster of four low power Hall thrusters show that the variations in discharge currents are related. This is believed to indicate that the thrusters are interacting with each other via cross talk through the plasma plumes. The most likely explanation for the synchronization of the current variations is believed to be related to changes in plasma resistivity as a result of small changes in electron number density.

Measurements of plasma properties in the thruster plumes have shown unexpectedly high electron temperatures along the centerline of each thruster. These hot spots are believed to be caused by collisionless shock waves. Such collisionless shocks can be produced by the ion-ion two-stream instability, which has been shown to be unstable along the thruster centerline. A simple geometric model has shown the relatively large changes in electron temperature measured with the triple probe to be consistent with conservation equations across a collisionless shock, even in the absence of a large change in plasma density. Additional measurements are needed to further understand the processes that occur along the centerline and to reach a definitive conclusion regarding the existence of collisionless shocks in Hall thruster plumes. Qualitative arguments have been presented indicating that collisionless shocks are unlikely to be caused by clustering multiple thrusters.

\section{$\underline{\text { References }}$}

1. Spanjers, G.G., et al., "The USAF Electric Propulsion Research Program," AIAA-2000$3146,36^{\text {th }}$ Joint Propulsion Conference \& Exhibit, Huntsville, AL, 2000.
2. Spores, R.A., et al., "Overview of the USAF Electric Propulsion Program," AIAA-2001$3225,37^{\text {th }}$ Joint Propulsion Conference \& Exhibit, Salt Lake City, UT, 2001.

3. Dunning Jr., J., et al., "NASA's Electric Propulsion Program," IEPC-01-002, $27^{\text {th }}$ International Electric Propulsion Conference, Pasadena, CA, 2001.

4. Hargus, W. and Reed, G., "The Air Force Clustered Hall Thruster Program," AIAA2002-3677, 38 ${ }^{\text {th }}$ Joint Propulsion Conference \& Exhibit, Indianapolis, IN, 2002.

5. Chen, S. and Sekiguchi, T., "Instantaneous Direct-Display System of Plasma Parameters by Means of Triple Probe," Journal of Applied Physics, V. 36, No. 8, 1965, pp. 2363-2375.

6. Haas, J.M., et al., "Hall Thruster Discharge Chamber Plasma Characterization Using a High-Speed Axial Reciprocating Electrostatic Probe," AIAA-99-2430, 35 ${ }^{\text {th }}$ Joint Propulsion Conference \& Exhibit, Los Angeles, CA, 1999.

7. Tilley, D.L., et al., "The Application of the Triple Probe Method to MPD Thruster Plumes," AIAA-90-2667, 21 $1^{\text {st }}$ International Electric Propulsion Conference, Orlando, FL, 1990.

8. Burton, R.L., et al., "Application of Multiple Electrostatic Probes to a Low Power Arcjet," AIAA-94-3299, $30^{\text {th }}$ Joint Propulsion Conference \& Exhibit, Indianapolis, IN 1994.

9. Hruby, V., et al., "Development of Low Power Hall Thrusters," AIAA-99-3534, $30^{\text {th }}$ Plasmadynamics and Lasers Conference, Norfolk, VA, 1999.

10. Haas, J.M., et al., "Performance Characteristics of a $5 \mathrm{~kW}$ Laboratory Hall Thruster," AIAA-98-3503, $34^{\text {th }}$ Joint Propulsion Conference \& Exhibit, Cleveland, $\mathrm{OH}, 1998$.

11. Goldston, R.J. and Rutherford, P.H., Introduction To Plasma Physics, Institute of Physics Publishing, Philadelphia, PA, 1997.

12. Williams, G.J., et al., "Correlating Laser Induced Fluorescence and Molecular Beam Mass Spectrometry Ion Energy Distributions," Journal of Propulsion and Power, V. 18, No. 2, 2002, pp. 489-490.

13. Williams, G.J., et al., "Laser Induced Fluorescence Measurement of Ion Velocities in the Plume of a Hall Effect Thruster," AIAA-99-2424, $35^{\text {th }}$ Joint Propulsion Conference \& Exhibit, Los Angeles, CA, 1999.

14. NRL Plasma Formulary, Naval Research Laboratory, Washington, DC, 1998. 
15. Nicholson, D.R., Introduction To Plasma Theory, John Wiley \& Sons, Inc., USA, 1983.

16. McKee, C.F., "Simulation of Counterstreaming Plasmas with Application to Collisionless Electrostatic Shocks," Physics Review Letters, V. 24, No. 18, 1970, pp. 990994.

17. Stringer, T.E., "Electrostatic Instabilities in Current-Carrying and Counterstreaming Plasmas," Plasma Physics, V. 6, 1964, pp. 267-279.

18. Papadopoulos, et al., "Heating of Counterstreaming Ion Beams in an External Magnetic Field," Physics of Fluids, V. 14, No. 4, 1971, pp. 849-857.

19. Gratton, F. and Gnavi, G., "Two-stream Instability in Convergent Geometry," Physics of Fluids, V. 30, No. 2, 1987, pp. 548-556.

20. Smith, T.B., et al., "Deconvolution of Axial Velocity Distributions from Hall Thruster LIF Spectra," IEPC-01-0019, 27 $7^{\text {th }}$ International Electric Propulsion Conference, Pasadena, CA, 2001.

21. Taylor, R.J., et al., "Observation of Collisionless Electrostatic Shocks," Physical Review Letters, V. 24, No. 5, 1970, pp. 206209.

22. Eselevich, V.G. and Fainshtein, V.G., "Turbulent Electrostatic Shock Wave in an Interaction of Oppositely Directed LowDensity Plasma Streams," Soviet Journal of Plasma Physics, V. 10, No. 3, 1984, pp. 313318.

23. Tidman, D.A. and Krall, N.A., Shock Waves in Collisionless Plasmas, John Wiley \& Sons, USA, 1971.
24. Chen, F.F., Introduction to Plasma Physics and Controlled Fusion, Plenum Press, New York, NY, 1984.

25. Kantrowitz, A. and Petschek, H., "MHD Characteristics and Shock Waves," in Plasma Physics in Theory and Application, ed. Kunkel, McGraw-Hill, USA, 1966.

26. Goodrich, C.C. and Scudder, J.D., "The Adiabatic Energy Change of Plasma Electrons and the Frame Dependence of the Cross-Shock Potential at Collisionless Magnetosonic Shock Waves," Journal of Geophysical Research, V. 89, No. A8, 1984, pp. 6654-6662.

27. Scudder, J.D., "A Review of the Physics of Electron Heating at Collisionless Shocks," Advances in Space Research, V. 15, No. 8/9, 1995, pp. 181-223.

28. Sanderson, J.J., "Jump Conditions Across a Collisionless, Perpendicular Shock," Journal of Physics D: Applied Physics, V. 9, 1976, pp. 2327-2330.

29. Morse, D.L., "Electrostatic Potential Rise Across Perpendicular Shocks," Plasma Physics, Vol. 15, 1973, pp. 1262-1264.

30. Schwartz, S.J., et al., "Electron Heating and the Potential Jump Across Fast Mode Shocks," Journal of Geophysical Research, V. 93, No. A11, 1988, pp. 12923-12931.

31. Gedalin, M., et al., "Electron Heating in Quasiperpendicular Shocks," Advances in Space Research, V. 15, No. 8/9, 1995, pp. 225-233. 